\section{Avaliação da qualidade da atenção pré-natal dentre gestantes com e sem história de prematuridade no Sistema Único de Saúde no Rio de Janeiro, Brasil}

\section{Assessment of quality of prenatal care among pregnant women with a history of premature deliveries using the Brazilian National Health Service in Rio de Janeiro, Brazil}

Marcelo Vianna Vettore 1

Marcos Dias 2

Mario Vianna Vettore 3

Maria do Carmo Leal 4
Abstract

Objectives: to assess the adequacy of prenatal care and the satisfaction and gestational risks of pregnant women with a history of premature deliveries.

Methods: a cross-sectional study with 1239 pregnant women, 259 with a history of premature deliveries when being attended by the Brazilian National Health Service (SUS) in RJ in 2007/2008. Demographic and socio-economic information, the obstetric history, adequacy of prenatal care and satisfaction with prenatal care were variables gathered using interviews and the prenatal records. The $\chi^{2}$ test was used to compare the group with the history of premature deliveries with the groups with low risk or no history of premature delivery.

Results: there were no differences in terms of the adequacy of prenatal health care among pregnant women with a history of premature deliveries compared with those with low gestational risk. Women were dissatisfied with the waiting times for consultations, the opening times of health units and the explanations given by health workers.

Conclusions: a history of premature delivery did not improve the quality of prenatal care for these pregnant women. The health units experienced a number of problems, in the view of the women, regarding their functioning. The explanations given by health workers regarding the risk of premature delivery did not meet the expectations of the women. Key words Infant, premature, Infant, low birth weight, Prenatal care, Health evaluation
1 Hospital Federal dos Servidores do Estado. Rua Sacadura Cabral, 178. Saúde. Rio de Janeiro, RJ, Brasil. CEP: 20.081-262. E-mail: marcelo.vettore@gmail.com

2 Instituto Fernandes Figueira. Fundação Oswaldo Cruz. Rio de Janeiro, RJ, Brasil.

${ }^{3}$ Instituto de Estudos em Saúde Coletiva. Universidade Federal do Rio de Janeiro. Rio de Janeiro, RJ, Brasil.

${ }^{4}$ Escola Nacional de Saúde Pública Sergio Arouca. Fundação Oswaldo Cruz. Rio de Janeiro, RJ, Brasil.

\section{Resumo}

Objetivos: avaliar adequação, acompanhamento pré-natal, satisfação e riscos gestacionais das gestantes com história de prematuridade.

Métodos: estudo seccional com 1239 gestantes, 259 com história de prematuridade no Sistema Único de Saúde (SUS) do RJ em 2007/2008. Informações demográficas, socio-econômicas, história obstétrica, adequação pré-natal e satisfação foram coletadas através de entrevistas e do cartão pré-natal. Utilizouse teste $\chi^{2}$ para comparar o grupo com história de prematuridade com os grupos de baixo risco e sem história de prematuridade.

Resultados: não houve diferenças na adequação $e$ no cuidado pré-natal entre as gestantes com história de prematuridade comparadas às de baixo risco gestacional. Houve insatisfação com tempo de espera pelas consultas, horário de funcionamento das unidades de saúde e explicações dos profissionais de saúde.

Conclusões: a presença de história de prematuridade não influenciou para que essas gestantes tivessem um pré-natal de melhor qualidade. As unidades de saúde apresentaram problemas, segundo a percepção das gestantes, em relação ao modo de funcionamento. As explicações dos profissionais de saúde sobre risco da prematuridade não atenderam às expectativas das gestantes.

Palavras-chave Prematuro, Recém-nascido de baixo peso, Cuidado pré-natal, Avaliação em saúde 


\section{Introdução}

A atenção pré-natal adequada é considerada uma das principais ações de promoção à saúde da gestante e do feto e prevenção de eventos adversos da gestação no âmbito da atenção primária à saúde. A melhoria do cuidado pré-natal pode ser considerada uma das mais importantes metas em termos de saúde pública devido à possibilidade de redução dos determinantes da morbimortalidade neonatal.

O Ministério da Saúde recomenda o início do pré-natal no primeiro trimestre e um mínimo de seis consultas, sendo uma no primeiro trimestre, duas no segundo e três no terceiro. Após o parto deve ser feita uma consulta até a $42^{\mathrm{a}}$ semana de puerperio. ${ }^{1}$

Mesmo com os esforços para melhorar a assistência ao pré-natal, ocorreu uma tendência no aumento na prevalência da prematuridade no Brasil a partir da década de 1990 , que atingiu até $15 \%$ nas Regiões Sul e Sudeste e 10\% no Nordeste. ${ }^{2}$ Houve um aumento da cobertura do pré-natal no país, mas não foi acompanhado da melhoria da qualidade da assistência às gestantes. Leal et al. ${ }^{3}$ verificaram $96 \%$ das puérperas haviam feito pré-natal, porém os índices de pré-natais inadequados ou intermediários foram predominantes, $57 \%$.

Os partos prematuros são, no Brasil e no mundo, a principal causa de morbimortalidade neonatal e pós-neonatal. ${ }^{4} \mathrm{Em}$ função destes aspectos, possíveis fatores de risco para a sua ocorrência têm sido investigados, e incluem características demográficas e socioeconômicas, comportamentos inadequados relacionados à saúde, fatores emocionais, doenças durante a gravidez, gemelaridade, história obstétrica e cuidados pré-natais inadequados. 5,6

Sayres et al. ${ }^{7}$ ressaltaram que história de parto prematuro foi o fator de risco mais importante para prematuridade e descreveram ainda outros fatores como intervalo intergestacional inferior a seis meses, infecções pélvicas e intrauterina, uso de drogas, tabagismo, história de conização, cirurgia de alta frequência, e abdominal da gestante. A lista inclui doenças maternas como hipertensão arterial, diabetes ou doenças da tireóide, gestação múltipla, polidrâmnio ou oligodrâmnio, encurtamento cervical $(<3 \mathrm{~cm})$, anomalias uterinas e sangramento vaginal devido à placenta prévia ou descolamento prematuro de placenta. ${ }^{7}$ Goldenberg et al. ${ }^{8}$ acrescentaram a esses fatores a raça/cor de pele preta, doença periodontal e índice de massa corporal (IMC) materno baixo.

A identificação desses fatores de risco seguida de uma atenção adequada no pré-natal é uma importante estratégia para a redução da prematuridade e estas mulheres deveriam receber uma assistência diferenciada com profissionais de saúde capacitados e em serviços de saúde de referência. Gama et al. ${ }^{9}$ concluíram que a assistência pré-natal mostrou ser uma política compensatória eficiente para a prevenção da prematuridade. Porém, a realidade brasileira é outra. Em 2006, apenas 55\% das gestantes no Brasil e 62\% no Rio de Janeiro tiveram sete ou mais consultas de pré-natal. Apesar do aumento da cobertura de pré-natal no Brasil entre 2000 e 2006, reconhece-se que somente a melhoria da sua qualidade poderá levar a uma redução da prematuridade, pois é um evento que tende a se repetir em gestações futuras. ${ }^{10,11}$

O impacto positivo do pré-natal na redução do risco para a prematuridade está relacionado ao diagnóstico de patologias e intervenção precoces como uso de progesterona, tratamento de infecções e realização de cerclagem do colo uterino para incompetência istmocervical.12,13

A maioria dos estudos sobre este tema abordou os determinantes e os efeitos sobre o recém-nascido prematuro, como hemorragia intraventricular, leucomalacia periventricular, displasia broncopulmonar, enterocolite necrotizante e deficiências cognitivas e motoras ao longo da vida.14,15 Porém, poucos estudos avaliaram a historia de prematuridade e fatores relacionados a ela.

É importante um melhor conhecimento da qualidade de atendimento às gestantes que já tiveram parto prematuro e a identificação de riscos gestacionais associados à prematuridade. Com isso será possível promover um aperfeiçoamento nas condutas durante o pré-natal, e, desta forma, ajudar a reduzir efetivamente as suas taxas. O objetivo deste estudo foi comparar as características sociodemográficas, obstétricas e a atenção da assistência pré-natal entre gestantes com história de prematuridade, gestantes sem esta história e gestantes de baixo risco no Sistema Único de Saúde (SUS) no município do Rio de Janeiro, Brasil.

\section{Métodos}

Foi desenvolvido um estudo seccional no período de outubro de 2007 a maio de 2008, com gestantes atendidas nos estabelecimentos do SUS do município do Rio de Janeiro. Esse fez parte de um estudo maior, cujo objetivo principal foi avaliar a "adequação da atenção pré-natal em gestantes no município do Rio de Janeiro". O tamanho da amostra foi estabelecido considerando-se este desfecho, estimado em $50 \%,{ }^{3}$ nível de significância de $5 \%$ e margem de erro bilateral de $2,5 \%$. Foi feita correção para população 
finita ( $\mathrm{n}=25.208$ consultas de pré-natal/mês) e efeito de desenho, estimado em 1,5. A amostra utilizada nesse estudo foi a mesma do estudo principal.

A amostragem foi feita por conglomerados em dois estágios. No primeiro estágio foram selecionados os estabelecimentos de saúde com atendimento pré-natal de baixo risco na rede SUS do município do Rio de Janeiro e estratificadas em: Unidades Básicas de Saúde (UBS), hospitais e casa de parto e tinham que apresentar média superior a 80 consultas de pré-natal por mês. Foram selecionados $40 \%$ dos hospitais $(n=5)$ e das UBS $(n=26)$ e como só existia uma casa de parto, a mesma foi selecionada.

Dentro dos estratos de UBS e hospitais, as unidades primárias de seleção foram estratificadas por área de planejamento e por divisão territorial e administrativa, conforme modelo adotado pela Secretaria Municipal de Saúde do Rio de Janeiro. 16 Fez-se seleção aleatória simples das unidades de saúde nos estratos de áreas de planejamento. Em cada uma dessas, o número de unidades selecionadas foi proporcional ao número de unidades de saúde existentes em todas as áreas.

O segundo estágio da amostragem selecionou as gestantes dentro dos estabelecimentos de saúde, conforme o turno de atendimento, sistematicamente, segundo ordem de saída das consultas de pré-natal, até completar a amostra prevista para cada unidade de saúde. Antes de serem iniciadas as consultas de pré-natal nas unidades selecionadas, os entrevistadores verificavam o número de gestantes que seriam atendidas no turno e dividiam o mesmo por seis (número de gestantes a serem entrevistadas por turno) e, assim, era obtido o intervalo para a seleção sistemática das mulheres.

A alocação da amostra por estratos foi feita proporcionalmente ao número de consultas pré-natal em cada estrato, sendo de $2 \%$ para a casa de parto, $63 \%$ para as UBS e $35 \%$ para os hospitais. O tamanho da amostra de cada estrato foi de 1313 nas UBS, 832 nos hospitais ou maternidades e 73 na casa de parto, totalizando 2218 gestantes.

Embora a casa de parto tenha sido considerada um estrato no planejamento da amostra, na fase de análise ela foi excluída, para permitir a inclusão do efeito de desenho na análise, dado que tem sido demonstrado o impacto de variáveis com a inclusão do plano de amostragem por conglomerado na precisão das estimativas. ${ }^{17}$ Para a realização da análise estatística, cada elemento da amostra recebeu uma ponderação pelo inverso de sua probabilidade de seleção e uma calibração para restituir a distribuição conhecida das consultas de pré-natal.
Os dados foram coletados a partir de informações obtidas do cartão pré-natal e de um questionário padronizado aplicado às gestantes selecionadas por entrevistadores previamente treinados, na própria unidade de saúde.

Todos os questionários foram revisados por médicos com experiência na assistência perinatal, codificados e armazenados no programa Windows Access (Microsoft Corp., Estados Unidos). A digitação foi realizada com dupla entrada em $100 \%$ dos questionários para avaliação da concordância e verificação de erros.

História de prematuridade foi definida como gestantes que informaram parto prematuro em gestação anterior e/ou se esta informação estava anotada no cartão do pré-natal. As gestantes de baixo risco eram as que não apresentaram hipertensão arterial, diabetes, história de prematuridade ou história de natimortalidade e/ou neomortalidade.

Foram incluídas as gestantes que possuíam o cartão de pré-natal e estavam com o cartão de prénatal no momento da entrevista e sabiam informar sobre a história de prematuridade. O critério de exclusão foi ser primípara.

As variáveis estudadas foram características demográficas e socioeconômicas: idade; raça/cor da pele; escolaridade; situação conjugal e atividade remunerada. A qualidade da assistência pré-natal foi avaliada com o Índice de Kotelchuck, considerandose: inadequado - realização de menos de $50 \%$ das consultas esperadas ou início do pré-natal após o $4^{\circ}$ mês de gestação; intermediário, adequado e mais do que adequado - com realização de 50-79\%; 80-109\% $\mathrm{e} \geq 110 \%$ das consultas esperadas, respectivamente. 18

A avaliação da história obstétrica e risco gestacional incluiu: número de gestações anteriores; estado nutricional pré-gravídico (baixo peso, eutrófica, sobrepeso ou obesa); idade gestacional no início do pré-natal (PN); idade materna de risco ( $\leq 19$ anos ou $\geq 35$ anos); hipertensão arterial; diabetes; sífilis; infecção urinária; anemia e história natimortalidade e/ou neomortalidade e baixo peso ao nascer. Foram verificadas nos cartões de pré-natal as anotações da idade gestacional, peso e pressão arterial em todas as consultas; fundo uterino e batimento cardíaco fetal após a $20^{\mathrm{a}}$ semana e os resultados de exames complementares da hemoglobina, Venereal Disease Research Laboratory (VDRL), anti-HIV, Elementos Anormais Sedimentados (EAS) e ultrassonografia obstétrica.

$\mathrm{Na}$ avaliação da satisfação das gestantes, se incluíram perguntas sobre o tempo de espera para ser atendida; maneira como foi tratada antes e durante a consulta; respeito a sua privacidade; explicações dos 
profissionais de saúde sobre riscos da prematuridade; horário de funcionamento da unidade de saúde e o que ela diria do atendimento pré-natal. As respostas foram categorizadas em ótimo, bom, regular, ruim ou péssimo.

Para análise estatística, inicialmente foram estimados os pesos amostrais por tipo de unidade de saúde. As variáveis coletadas foram categorizadas. A associação entre história de prematuridade e riscos gestacionais, preenchimento do cartão de pré-natal e satisfação com o pré-natal do SUS foi avaliada através do teste qui-quadrado, conside-rando-se o nível de significância de 5\%. Utilizou-se o software estatístico SPSS 17.0 (SPSS Inc., Chicago, Estados Unidos) em todas as análises de dados.

O presente estudo foi aprovado pelo Comitê de Ética em Pesquisa da Escola Nacional de Saúde Pública Sergio Arouca, Fundação Oswaldo Cruz (ENSP/FIOCRUZ) (Parecer $\mathrm{n}^{\circ}$ 142/06). Foi entregue um Termo de Consentimento Livre e Esclarecido às participantes ou ao responsável por menores de idade.

\section{Resultados}

Foram convidadas 2331 gestantes, das quais 92 $(3,9 \%)$ recusaram participar, $923(39,6 \%)$ foram excluídas porque eram primigestas e $77(3,3 \%)$ porque não sabiam informar história de prematuridade. A amostra final foi composta por 1239 gestantes, das quais 259 (20,9\%) tinham história de prematuridade e $980(79,1 \%)$ não. O grupo de baixo risco foi composto por 856 gestantes: 980 sem história de prematuridade menos 124 que não apresentaram hipertensão arterial, diabetes ou história de natimortalidade e/ou neomortalidade.

A maioria das participantes tinha entre 20 e 34 anos de idade, era da cor de pele parda, possuía até oito anos de estudo, vivia com companheiro e não tinha atividade remunerada. A proporção de mulheres de cor de pele preta, com atividade remunerada e com três ou mais gestações anteriores foi estatisticamente maior entre aquelas com história de prematuridade em relação às de baixo risco. Quase $70 \%$ das participantes foram classificadas na categoria de pré-natal adequado, enquanto que para $14 \%$ observou-se inadequação (Tabela 1).

Informações sobre o peso e a pressão arterial foram as mais registradas no cartão de pré-natal. Tiveram anotações intermediárias, entre 50 e $89 \%$, o fundo uterino, batimento cardíaco fetal, a idade gestacional, VDRL, ultrassonografia no terceiro trimestre e EAS. Menos da metade dos cartões de pré-natal tinha registro do anti-HIV $(46,8 \%)$ e ultrassonografia no primeiro trimestre $(42,7 \%)$. A maioria das participantes realizou entre uma e três ultrassonografias obstétricas e fez esse exame no $1^{\circ}$ e $2^{\circ}$ trimestres. Nenhuma das informações registradas no cartão da gestante e sobre o exame de ultrassonografia obstétrica foi estatisticamente diferentes entre as gestantes com e sem história de prematuridade (Tabela 2).

Enquanto mais de $80 \%$ das gestantes relataram boa ou ótima satisfação em relação à maneira como foram tratadas antes e durante as consultas e o respeito a sua privacidade, o tempo de espera de espera foi considerado bom ou ótimo por apenas $41,6 \%$ delas. A boa ou ótima satisfação em relação a explicações do profissional de saúde, horário de funcionamento e a avaliação geral do atendimento pré-natal variou de $61,9 \%$ a $73,4 \%$. A pior satisfação com o tempo de espera para ser atendida e as explicações dos profissionais de saúde foram significativamente maiores entre as gestantes com história de prematuridade em comparação com as de baixo risco (Tabela 3).

A proporção de gestantes com hipertensão arterial, com história de natimortalidade e/ou neomortalidade e história de recém-nascido (RN) com baixo peso ao nascer foi maior entre as com história de prematuridade em comparação aquelas sem esse passado obstétrico. A frequência de hipertensão arterial foi 1,7 vezes maior $(14,8 \% v s 8,8 \%, p<0,001)$ entre aquelas com história de parto prematuro em comparação com as sem esse histórico; aquelas com história de baixo peso ao nascer foi 9,6 vezes maior $(44,3 \%$ vs $4,6 \%, p<0,001)$ e com história de natimortalidade e/ou neomortalidade foi 9,7 vezes maior $(26,3 \%$ vs $2,7 \%, p<0,001)$. Por outro lado, a diabetes foi mais comum entre as gestantes sem história de prematuridade em relação àquelas com essa história $(p=0,01)$, mas só havia informações disponíveis para $37,7 \%$ do total de gestantes (Tabela 4 ). 
Perfil sociodemográfico e obstétrico das gestantes no pré-natal do Sistema Único de Saúde, segundo a presença ou ausência de história de prematuridade. Município do Rio de Janeiro, Brasil, 2007-2008.

\begin{tabular}{|c|c|c|c|c|c|c|c|}
\hline \multirow[t]{2}{*}{ Características das gestantes } & \multicolumn{2}{|c|}{$\begin{array}{l}\text { História de prematuridade } \\
\qquad(\mathrm{N}=259)\end{array}$} & \multicolumn{2}{|c|}{$\begin{array}{l}\text { Baixo risco } \\
(\mathrm{N}=856)\end{array}$} & \multicolumn{2}{|c|}{$\begin{array}{c}\text { Total } \\
(\mathrm{N}=1115)\end{array}$} & \multirow[t]{2}{*}{$p^{*}$} \\
\hline & $\mathrm{n}$ & $\%$ & $\mathrm{n}$ & $\%$ & $\mathrm{n}$ & $\%$ & \\
\hline \multicolumn{8}{|l|}{ Idade da gestante (anos) } \\
\hline$\leq 19$ & 19 & 7,3 & 80 & 9,4 & 99 & 8,9 & \\
\hline 20 a 34 & 205 & 79,2 & 679 & 79,4 & 884 & 79,4 & 0,42 \\
\hline$\geq 35$ & 35 & 13,5 & 96 & 11,2 & 131 & 11,8 & \\
\hline Total & 259 & 100,0 & 855 & 100,0 & 1114 & 100,0 & \\
\hline \multicolumn{8}{|l|}{ Cor da pele** } \\
\hline Branca & 61 & 24,2 & 212 & 25,4 & 273 & 25,1 & \\
\hline Parda & 120 & 47,6 & 446 & 53,3 & 566 & 52,0 & 0,07 \\
\hline Preta & 71 & 28,2 & 178 & 21,3 & 249 & 22,9 & \\
\hline Total & 252 & 100,0 & 836 & 100,0 & $1088 * *$ & 100,0 & \\
\hline \multicolumn{8}{|l|}{ Anos de estudo } \\
\hline$\leq 8$ & 139 & 53,9 & 448 & 52,3 & 587 & 52,7 & \\
\hline$>8$ & 119 & 46,1 & 408 & 47,7 & 527 & 47,3 & 0,51 \\
\hline Total & 258 & 100,0 & 586 & 100,0 & 1114 & 100,0 & \\
\hline \multicolumn{8}{|l|}{ Situação conjugal } \\
\hline Não vive com o companheiro & 48 & 18,5 & 131 & 15,3 & 179 & 16,1 & \\
\hline Vive com companheiro & 211 & 81,5 & 724 & 84,7 & 935 & 83,9 & 0,23 \\
\hline Total & 259 & 100,0 & 855 & 100,0 & 1114 & 100,0 & \\
\hline \multicolumn{8}{|l|}{ Atividade remunerada } \\
\hline Sim & 112 & 43,2 & 290 & 33,9 & 402 & 36,1 & \\
\hline Não & 147 & 56,8 & 565 & 66,1 & 712 & 63,9 & 0,02 \\
\hline Total & 259 & 100,0 & 855 & 100,0 & 1114 & 100,0 & \\
\hline \multicolumn{8}{|l|}{ Número de gestações anteriores } \\
\hline 1 ou 2 & 143 & 55,2 & 650 & 75,9 & 793 & 71,1 & \\
\hline$\geq 3$ & 116 & 44,8 & 206 & 24,1 & 322 & 28,9 & $<0,001$ \\
\hline Total & 259 & 100,0 & 856 & 100,0 & 1115 & 100,0 & \\
\hline \multicolumn{8}{|l|}{ Estado nutricional pré-gestacional } \\
\hline Baixo peso & 37 & 17,8 & 93 & 14,6 & 130 & 15,4 & \\
\hline Eutrófica & 91 & 43,8 & 269 & 42,3 & 360 & 42,7 & \\
\hline Sobrepeso & 48 & 23,1 & 181 & 28,5 & 229 & 27,1 & 0,51 \\
\hline Obesa & 32 & 15,3 & 93 & 14,6 & 125 & 14,8 & \\
\hline Total & 208 & 100,0 & 636 & 100,0 & 844 & 100,0 & \\
\hline \multicolumn{8}{|c|}{ Idade gestacional no início do pré-natal } \\
\hline $1^{\circ}$ trimestre & 134 & 57,5 & 457 & 59,9 & 591 & 59,3 & \\
\hline $2^{\circ}$ trimestre & 96 & 41,2 & 284 & 37,2 & 380 & 38,2 & 0,51 \\
\hline $3^{\circ}$ trimestre & 3 & 1,3 & 22 & 2,9 & 25 & 2,5 & \\
\hline Total & 233 & 100,0 & 763 & 100,0 & 996 & 100,0 & \\
\hline \multicolumn{8}{|l|}{ Índice de Kotelchuck } \\
\hline Mais que adequado & 18 & 7,7 & 53 & 6,9 & 71 & 7,1 & \\
\hline Adequado & 162 & 69,3 & 533 & 69,8 & 695 & 69,7 & \\
\hline Parcialmente adequado & 23 & 9,8 & 71 & 9,3 & 94 & 9,4 & 0,96 \\
\hline Inadequado & 31 & 13,2 & 107 & 14,0 & 138 & 13,8 & \\
\hline Total & 234 & 100,0 & 764 & 100,0 & 998 & 100,0 & \\
\hline
\end{tabular}

* qui-quadrado; ** excluídas 27 (amarelas e indígenas). 
Tabela 2

Resultados informados no cartão de pré-natal das gestantes do Sistema Único de Saúde segundo a presença ou ausência de história de prematuridade. Município do Rio de Janeiro, Brasil, 2007-2008.

\begin{tabular}{|c|c|c|c|c|c|c|c|}
\hline \multirow[t]{2}{*}{ Características das gestantes } & \multicolumn{2}{|c|}{$\begin{array}{l}\text { História de prematuridade } \\
\qquad(\mathrm{N}=259)\end{array}$} & \multicolumn{2}{|c|}{$\begin{array}{l}\text { Baixo risco } \\
(\mathrm{N}=856)\end{array}$} & \multicolumn{2}{|c|}{$\begin{array}{c}\text { Total } \\
(\mathrm{N}=1115)\end{array}$} & \multirow[t]{2}{*}{$p^{*}$} \\
\hline & $\mathrm{n}$ & $\%$ & $\mathrm{n}$ & $\%$ & $\mathrm{n}$ & $\%$ & \\
\hline \multicolumn{8}{|c|}{$\begin{array}{l}\text { Anotação da idade gestacional no } \\
\text { cartão pré-natal }\end{array}$} \\
\hline $\operatorname{Sim}$ & 182 & 70,8 & 558 & 66,1 & 740 & 67,2 & 0,19 \\
\hline Total & 257 & 100,0 & 844 & 100,0 & 1101 & 100,0 & \\
\hline \multicolumn{8}{|c|}{$\begin{array}{l}\text { Peso anotado no cartão pré-natal em } \\
\text { todas as consultas }\end{array}$} \\
\hline $\operatorname{sim}$ & 245 & 95,0 & 808 & 95,6 & 1053 & 95,5 & 0,66 \\
\hline Total & 258 & 100,0 & 845 & 100,0 & 1103 & 100,0 & \\
\hline \multicolumn{8}{|c|}{$\begin{array}{l}\text { Pressão arterial anotada no cartão } \\
\text { pré-natal em todas as consultas }\end{array}$} \\
\hline $\operatorname{Sim}$ & 246 & 95,0 & 811 & 94,9 & 1057 & 94,9 & 0,89 \\
\hline Total & 259 & 100,0 & 855 & 100,0 & 1114 & 100,0 & \\
\hline \multicolumn{8}{|c|}{$\begin{array}{l}\text { Fundo uterino anotado no cartão } \\
\text { pré-natal em todas as consultas com } \\
\text { IG }>20 \text { semanas }\end{array}$} \\
\hline Sim & 168 & 87,5 & 575 & 85,6 & 743 & 86,0 & 0,59 \\
\hline Total & 192 & 100,0 & 672 & 100,0 & 864 & 100,0 & \\
\hline \multicolumn{8}{|c|}{$\begin{array}{l}\text { Anotação do bcf no cartão pré-natal } \\
\text { (IG>20 semanas) }\end{array}$} \\
\hline $\operatorname{sim}$ & 155 & 80,7 & 531 & 79,0 & 686 & 79,4 & 0,65 \\
\hline Total & 192 & 100,0 & 672 & 100,0 & 864 & 100,0 & \\
\hline \multicolumn{8}{|c|}{$\begin{array}{l}\text { Anotação da hemoglobina no cartão } \\
\text { de pré-natal }\end{array}$} \\
\hline $\operatorname{sim}$ & 142 & 75,5 & 436 & 74,7 & 578 & 74,9 & 0,75 \\
\hline Total & 188 & 100,0 & 584 & 100,0 & 772 & 100,0 & \\
\hline \multicolumn{8}{|c|}{ Anotação de VDRL no cartão de pré-natal } \\
\hline $\operatorname{sim}$ & 141 & 54,7 & 531 & 62,8 & 672 & 60,9 & 0,10 \\
\hline Total & 258 & 100,0 & 846 & 100,0 & 1104 & 100,0 & \\
\hline \multicolumn{8}{|c|}{$\begin{array}{l}\text { Anotação de anti-HIV no cartão de } \\
\text { pré-natal }\end{array}$} \\
\hline $\operatorname{sim}$ & 109 & 42,2 & 407 & 48,2 & 516 & 46,8 & 0,19 \\
\hline Total & 258 & 100,0 & 36,9 & 100,0 & 1103 & 100,0 & \\
\hline \multicolumn{8}{|c|}{ Anotação de EAS no cartão de pré-natal } \\
\hline $\operatorname{sim}$ & 63 & 54,3 & 263 & 58,1 & 326 & 57,3 & 0,61 \\
\hline Total & 116 & 100,0 & 453 & 100,0 & 569 & 100,0 & \\
\hline \multicolumn{8}{|c|}{ Realização de ultrassonografia obstétrica } \\
\hline Não fez nenhuma & 5 & 3,0 & 20 & 3,4 & 25 & 3,3 & \\
\hline Fez entre 1 e 3 & 154 & 91,1 & 535 & 91,3 & 689 & 91,3 & 0,93 \\
\hline Fez mais de 3 & 10 & 5,9 & 31 & 5,3 & 41 & 5,4 & \\
\hline Total & 169 & 100,0 & 586 & 100,0 & 755 & 100,0 & \\
\hline
\end{tabular}

* qui-quadrado; bcf=batimento cardíaco fetal; IG= idade gestacional; VDRL= Venereal Disease Research Laboratory; EAS= Elementos Anormais Sedimentados. 
Resultados informados no cartão de pré-natal das gestantes do Sistema Único de Saúde segundo a presença ou ausência de história de prematuridade. Município do Rio de Janeiro, Brasil, 2007-2008.

\begin{tabular}{|c|c|c|c|c|c|c|c|}
\hline \multirow[t]{2}{*}{ Características das gestantes } & \multicolumn{2}{|c|}{$\begin{array}{l}\text { História de prematuridade } \\
\qquad(\mathrm{N}=259)\end{array}$} & \multicolumn{2}{|c|}{$\begin{array}{l}\text { Baixo risco } \\
(\mathrm{N}=856)\end{array}$} & \multicolumn{2}{|c|}{$\begin{array}{c}\text { Total } \\
(\mathrm{N}=1115)\end{array}$} & \multirow[t]{2}{*}{$p^{*}$} \\
\hline & $\mathrm{n}$ & $\%$ & $\mathrm{n}$ & $\%$ & $\mathrm{n}$ & $\%$ & \\
\hline \multicolumn{8}{|l|}{$\begin{array}{l}\text { Realização de ultrassonografia } \\
\text { obstétrica }\end{array}$} \\
\hline \multicolumn{8}{|l|}{$1^{\circ}$ trimestre } \\
\hline $\operatorname{Sim}$ & 64 & 42,1 & 218 & 42,9 & 282 & 42,7 & \\
\hline Não & 28 & 18,4 & 89 & 17,5 & 117 & 17,7 & 0,85 \\
\hline Não se aplica & 60 & 39,5 & 201 & 39,6 & 261 & 39,5 & \\
\hline Total & 152 & 100,0 & 508 & 100,0 & 660 & 100,0 & \\
\hline \multicolumn{8}{|l|}{$2^{\circ}$ trimestre } \\
\hline $\operatorname{Sim}$ & 116 & 82,3 & 395 & 84,0 & 511 & 83,7 & \\
\hline Não & 22 & 15,6 & 60 & 12,8 & 82 & 13,4 & 0,68 \\
\hline Não se aplica & 3 & 2,1 & 15 & 3,2 & 18 & 2,9 & \\
\hline Total & 141 & 100,0 & 470 & 100,0 & 611 & 100,0 & \\
\hline \multicolumn{8}{|l|}{$3^{\circ}$ trimestre } \\
\hline Sim & 29 & 56,9 & 136 & 62,1 & 165 & 61,1 & \\
\hline Não & 20 & 39,2 & 81 & 37,0 & 101 & 37,4 & 0,56 \\
\hline Não se aplica & 2 & 3,9 & 2 & 0,9 & 4 & 1,5 & \\
\hline Total & 51 & 100,0 & 219 & 100,0 & 270 & 100,0 & \\
\hline
\end{tabular}

* qui-quadrado; bcf=batimento cardíaco fetal; $I G=$ idade gestacional; VDRL= Venereal Disease Research Laboratory; EAS= Elementos Anormais Sedimentados.

Tabela 3

Satisfação com o pré-natal do Sistema Único de Saúde, segundo a presença ou ausência de história de prematuridade. Município do Rio de Janeiro, Brasil, 2007-2008.

\begin{tabular}{|c|c|c|c|c|c|c|c|}
\hline \multirow[t]{2}{*}{ Satisfação } & \multicolumn{2}{|c|}{$\begin{array}{l}\text { História de prematuridade } \\
\qquad(\mathrm{N}=259)\end{array}$} & \multicolumn{2}{|c|}{$\begin{array}{l}\text { Baixo risco } \\
(\mathrm{N}=856)\end{array}$} & \multicolumn{2}{|c|}{$\begin{array}{c}\text { Total } \\
(\mathrm{N}=1115)\end{array}$} & \multirow[t]{2}{*}{$p^{*}$} \\
\hline & $\mathrm{n}$ & $\%$ & $\mathrm{n}$ & $\%$ & $\mathrm{n}$ & $\%$ & \\
\hline \multicolumn{8}{|c|}{$\begin{array}{l}\text { Percepção da gestante sobre o tempo } \\
\text { de espera para atendimento }\end{array}$} \\
\hline Ótimo/ Bom & 95 & 36,7 & 369 & 43,1 & 464 & 41,6 & \\
\hline Regular & 75 & 29,0 & 281 & 32,9 & 356 & 32,0 & 0,02 \\
\hline Ruim / Péssimo & 89 & 34,3 & 205 & 24,0 & 294 & 26,4 & \\
\hline Total & 259 & 100,0 & 855 & 100,0 & 1114 & 100,0 & \\
\hline \multicolumn{8}{|c|}{$\begin{array}{l}\text { Percepção da gestante de como foi } \\
\text { tratada antes da consulta }\end{array}$} \\
\hline Ótimo/ Bom & 221 & 85,3 & 745 & 87,0 & 966 & 86,7 & \\
\hline Regular & 30 & 11,6 & 94 & 11,0 & 124 & 11,1 & 0,66 \\
\hline Ruim / Péssimo & 8 & 3,1 & 17 & 2,0 & 25 & 2,2 & \\
\hline \multirow[t]{2}{*}{ Total } & 259 & 100,0 & 856 & 100,0 & 1115 & 100,0 & \\
\hline & & & & & & & continua \\
\hline
\end{tabular}


Satisfação com o pré-natal do Sistema Único de Saúde, segundo a presença ou ausência de história de prematuridade. Município do Rio de Janeiro, Brasil, 2007-2008.

\begin{tabular}{|c|c|c|c|c|c|c|c|}
\hline \multirow[t]{2}{*}{ Satisfação } & \multicolumn{2}{|c|}{$\begin{array}{l}\text { História de prematuridade } \\
\qquad(\mathrm{N}=259)\end{array}$} & \multicolumn{2}{|c|}{$\begin{array}{l}\text { Baixo risco } \\
(N=856)\end{array}$} & \multicolumn{2}{|c|}{$\begin{array}{c}\text { Total } \\
(\mathrm{N}=1115)\end{array}$} & \multirow[t]{2}{*}{$p^{*}$} \\
\hline & $\mathrm{n}$ & $\%$ & $\mathrm{n}$ & $\%$ & $\mathrm{n}$ & $\%$ & \\
\hline \multicolumn{8}{|c|}{$\begin{array}{l}\text { Percepção da gestante de como foi } \\
\text { tratada durante a consulta }\end{array}$} \\
\hline Ótimo/ Bom & 205 & 79,5 & 713 & 83,3 & 918 & 82,4 & \\
\hline Regular & 37 & 14,3 & 106 & 12,4 & 143 & 12,8 & 0,28 \\
\hline Ruim / Péssimo & 16 & 6,2 & 37 & 4,3 & 53 & 4,8 & \\
\hline Total & 258 & 100,0 & 856 & 100,0 & 1114 & 100,0 & \\
\hline \multicolumn{8}{|c|}{ Respeito à privacidade } \\
\hline Ótimo/ Bom & 227 & 87,7 & 749 & 87,6 & 976 & 87,6 & \\
\hline Regular & 19 & 7,3 & 89 & 10,4 & 108 & 9,7 & 0,10 \\
\hline Ruim / Péssimo & 13 & 5,0 & 17 & 2,0 & 30 & 2,7 & \\
\hline Total & 259 & 100,0 & 855 & 100,0 & 1114 & 100,0 & \\
\hline \multicolumn{8}{|c|}{ Explicações dos profissionais de saúde } \\
\hline Ótimo/ Bom & 142 & 54,8 & 548 & 64,0 & 690 & 61,9 & \\
\hline Regular & 68 & 26,3 & 219 & 25,6 & 287 & 25,7 & 0,01 \\
\hline Ruim / Péssimo & 49 & 18,9 & 89 & 10,4 & 138 & 12,4 & \\
\hline Total & 259 & 100,0 & 856 & 100,0 & 1115 & 100,0 & \\
\hline \multicolumn{8}{|c|}{$\begin{array}{l}\text { Horário de funcionamento da unidade } \\
\text { de saúde }\end{array}$} \\
\hline Ótimo/ Bom & 172 & 66,7 & 586 & 69 & 758 & 68,5 & \\
\hline Regular & 40 & 15,5 & 155 & 18,3 & 195 & 17,6 & 0,20 \\
\hline Ruim / Péssimo & 46 & 17,8 & 108 & 12,7 & 154 & 13,9 & \\
\hline Total & 258 & 100,0 & 849 & 100,0 & 1107 & 100,0 & \\
\hline \multicolumn{8}{|c|}{$\begin{array}{l}\text { Impressão sobre do atendimento } \\
\text { pré-natal }\end{array}$} \\
\hline Ótimo/ Bom & 184 & 71 & 632 & 74,1 & 816 & 73,4 & \\
\hline Regular & 54 & 20,9 & 168 & 19,7 & 222 & 20,0 & 0,62 \\
\hline Ruim / Péssimo & 21 & 8,1 & 53 & 6,2 & 74 & 6,6 & \\
\hline Total & 259 & 100,0 & 853 & 100,0 & 1112 & 100,0 & \\
\hline
\end{tabular}

* qui-quadrado. 
Risco gestacional no pré-natal do Sistema Único de Saúde, segundo a presença ou ausência de história de prematuridade. Município do Rio de Janeiro, Brasil, 2007-2008.

\begin{tabular}{|c|c|c|c|c|c|c|c|}
\hline \multirow[t]{2}{*}{ Risco gestacional } & \multicolumn{2}{|c|}{$\begin{array}{l}\text { História de prematuridade } \\
\qquad(\mathrm{N}=259)\end{array}$} & \multicolumn{2}{|c|}{$\begin{array}{l}\text { Baixo risco } \\
(\mathrm{N}=856)\end{array}$} & \multicolumn{2}{|c|}{$\begin{array}{c}\text { Total } \\
(\mathrm{N}=1115)\end{array}$} & \multirow[t]{2}{*}{$p^{*}$} \\
\hline & $\mathrm{n}$ & $\%$ & $\mathrm{n}$ & $\%$ & $\mathrm{n}$ & $\%$ & \\
\hline \multicolumn{8}{|l|}{ Idade $<19$ anos } \\
\hline Sim & 19 & 7,3 & 88 & 9,0 & 107 & 8,6 & \\
\hline Não & 240 & 92,7 & 892 & 91,0 & 1132 & 91,4 & 0,34 \\
\hline Total & 259 & 100,0 & 980 & 100,0 & 1239 & 100,0 & \\
\hline \multicolumn{8}{|l|}{ Idade $\geq 35$ anos } \\
\hline Sim & 33 & 12,7 & 118 & 12,0 & 151 & 12,2 & \\
\hline Não & 226 & 87,3 & 862 & 88,0 & 1088 & 87,8 & 0,33 \\
\hline Total & 259 & 100,0 & 980 & 100,0 & 1239 & 100,0 & \\
\hline \multicolumn{8}{|c|}{ Hipertensão arterial } \\
\hline Sim & 38 & 14,8 & 84 & 8,8 & 122 & 10,0 & \\
\hline $\mathrm{N}$ ão & 219 & 85,2 & 874 & 91,2 & 1093 & 90,0 & $<0,001$ \\
\hline Total & 257 & 100,0 & 958 & 100,0 & 1215 & 100,0 & \\
\hline \multicolumn{8}{|l|}{ Diabetes } \\
\hline Sim & 2 & 2,2 & 14 & 3,7 & 16 & 3,4 & \\
\hline Não & 90 & 97,8 & 360 & 96,3 & 450 & 96,6 & 0,01 \\
\hline Total & 92 & 100,0 & 374 & 100,0 & 466 & 100,0 & \\
\hline \multicolumn{8}{|l|}{ Sífilis } \\
\hline Sim & 6 & 6,0 & 14 & 3,1 & 20 & 3,6 & \\
\hline Não & 94 & 94,0 & 436 & 96,9 & 530 & 96,4 & 0,34 \\
\hline Total & 100 & 100,0 & 450 & 100,0 & 550 & 100,0 & \\
\hline \multicolumn{8}{|l|}{ Infecção urinária } \\
\hline $\operatorname{Sim}$ & 56 & 51,4 & 213 & 44,7 & 269 & 45,9 & \\
\hline Não & 53 & 48,6 & 264 & 55,3 & 317 & 54,1 & 0,32 \\
\hline Total & 109 & 100,0 & 477 & 100,0 & 586 & 100,0 & \\
\hline \multicolumn{8}{|l|}{ Anemia } \\
\hline Sim & 54 & 42,2 & 202 & 36,8 & 256 & 37,8 & \\
\hline N ão & 74 & 57,8 & 347 & 63,2 & 421 & 62,2 & 0,18 \\
\hline Total & 128 & 100,0 & 549 & 100,0 & 677 & 100,0 & \\
\hline \multicolumn{8}{|c|}{$\begin{array}{l}\text { História de natimortalidade e/ou } \\
\text { neomortalidade }\end{array}$} \\
\hline Sim & 67 & 26,3 & 26 & 2,7 & 93 & 7,6 & \\
\hline Não & 188 & 73,7 & 944 & 97,3 & 1132 & 92,4 & $<0,001$ \\
\hline Total & 255 & 100,0 & 970 & 100,0 & 1225 & 100,0 & \\
\hline \multicolumn{8}{|c|}{ História de baixo peso ao nascer } \\
\hline Sim & 113 & 44,3 & 43 & 4,6 & 156 & 13,2 & \\
\hline Não & 142 & 55,7 & 882 & 95,4 & 1024 & 86,8 & $<0,001$ \\
\hline Total & 255 & 100,0 & 925 & 100,0 & 1180 & 100,0 & \\
\hline \multicolumn{8}{|c|}{ Estado nutricional pré-gestacional } \\
\hline Baixo peso & 37 & 17,8 & 98 & 13,5 & 135 & 14,5 & \\
\hline Eutrófica & 91 & 43,7 & 286 & 39,6 & 377 & 40,5 & \\
\hline Sobrepeso & 48 & 23,1 & 208 & 28,8 & 256 & 27,5 & 0,18 \\
\hline Obesa & 32 & 15,4 & 131 & 18,1 & 163 & 17,5 & \\
\hline Total & 208 & 100,0 & 723 & 100,0 & 931 & 100,0 & \\
\hline
\end{tabular}

* qui-quadrado. 


\section{Discussão}

Entre as gestantes com história de prematuridade, o mais frequente foi aquelas com atividade remunerada, comparadas às de baixo risco. Há muito tempo se questiona se as atividades laborais causam ou predispõe a prematuridade. Silva et al. ${ }^{19}$ não verificaram associação de trabalho materno na posição ortostática durante a gravidez e prematuridade. É possível que repouso no leito possa diminuir o risco de parto prematuro, mas não foi encontrada evidência que sustente essa associação. ${ }^{20}$ Esta conclusão pode ser mascarada pela complexa multicausalidade da prematuridade e seus fatores interrelacionados. Especialistas acreditam que esta prática não deva ser padronizada como parte do tratamento para prevenir prematuridade. 21

As multíparas predominaram no grupo de gestantes com história de prematuridade em relação às de baixo risco. A história de prematuridade tende a ser mais frequente quanto maior a possibilidade dela ocorrer, ou seja, quanto maior a paridade da paciente. Outro fator a ser considerado é a idade da gestante, pois as multíparas proporcionalmente se concentraram mais no grupo com mais idade, 35 anos ou mais, e as com menos filhos entre as adolescentes. Andrade et al.22 observaram que gestantes com mais de 39 anos de idade tiveram maior taxa de prematuridade em comparação àquelas com idade entre 18 e 29 anos, assim como maior ocorrência de baixo peso ao nascer e óbito fetal.

$\mathrm{Na}$ comparação entre os grupos ocorreu uma tendência de predomínio de mulheres de cor de pele preta, em relação às brancas e pardas, naquelas com história de prematuridade em comparação com as de baixo risco. Leal et al. ${ }^{23}$ verificaram que a qualidade da assistência pré-natal das gestantes de cor de pele preta foi pior, segundo índice de Kotelchuck, do que as de cor branca. Whitehead e Helms ${ }^{24}$ observaram, entre gestantes de baixo risco, chance maior de prematuridade nas gestantes de cor de pele preta em comparação às brancas, mas apenas entre multíparas.

As pacientes de risco devido ao histórico de prematuridade deveriam mas não receberam, atenção pré-natal diferenciada em relação às de baixo risco. Os registros nos cartões de pré-natal sobre a gravidez, exame físico e exames complementares não apresentaram diferenças entre esses dois grupos, assim como também não houve diferença na qualidade do pré-natal. Não apenas a presença, mas também a qualidade do pré-natal está diretamente relacionada ao risco de prematuridade. Vettore et al.5 identificaram associação entre número reduzido de consultas pré-natal com risco aumentado para parto prematuro. Beeckman et al. 25 utilizaram na avaliação de pré-natal o Content and Timing of care in Pregnancy, que leva em consideração a idade gestacional no início do pré-natal, a realização e o momento dos exames de sangue, ultrassonografia e verificação de pressão arterial. Esses autores observaram um risco menor de parto prematuro para as gestantes que se encontravam nas categorias de realização de pré-natal suficiente e apropriado comparadas àquelas do grupo considerado inadequado. Assunção et al. 26 também verificaram um maior risco para parto prematuro nas gestantes com pré-natal inadequado. Nessa avaliação os parâmetros utilizados foram: idade gestacional do início do pré-natal, número de consultas, exame de urina e de sangue, ultrassonografia, informações do exame físico e orientações às gestantes.

Entre as pacientes com história de prematuridade, a maioria recebeu solicitação de ultrassonografia obstétrica na primeira consulta de pré-natal e em apenas um quarto delas foi realizado toque vaginal. A medição do comprimento do colo uterino se mostrou muito relevante na predição da prematuridade, porém essa avaliação não foi realizada em $25 \%$ das gestantes, o que denota uma necessidade da melhoria da qualidade assistencial. Entre as medidas a serem adotadas no pré-natal estão a avaliação do comprimento cervical e o teste da fibronectina fetal, que demonstraram serem fatores preditores para a prematuridade. 27,28

As gestantes, de uma forma geral, estavam satisfeitas com o atendimento do profissional de saúde. As gestantes com história de prematuridade estavam descontentes com os profissionais de saúde em relação às explicações sobre riscos envolvidos na prematuridade e com a forma de funcionamento do serviço de saúde, em comparação com as sem esse passado obstétrico. Isso parece demonstrar que elas eram mais ansiosas e tiveram uma percepção pior em relação a esses aspectos. Mulheres com antecedentes de aborto espontâneo recorrente, óbito fetal, prematuridade ou óbito neonatal precoce parecem ter mais sintomas de ansiedade e depressão durante a gestação.6,29

Houve uma forte tendência de a hipertensão arterial estar associada à história de prematuridade em comparação ao grupo sem esse passado obstétrico. A hipertensão arterial pode acarretar complicações maternas e/ou fetais que podem levar a interrupção prematura da gravidez. As síndromes hipertensivas da gravidez foram associadas a um aumento no risco de parto prematuro.6,30

Recomenda-se, após os resultados apresentados neste artigo, que os serviços de pré-natal promovam educação continuada dos profissionais de saúde com 
enfoque na comunicação com o paciente, no preenchimento e valorização do cartão pré-natal como instrumento para identificar as gestantes de risco. Em relação às unidades de saúde sugere-se adequar o número de atendimentos no pré-natal, a fim de possibilitar um melhor tempo para esclarecimentos dos riscos da prematuridade na gravidez. É importante também chamar a atenção das chefias das unidades de saúde para criar instrumentos a fim de diminuir o tempo de espera pelas consultas de pré-natal e, na medida do possível, flexibilizar o horário de funcionamento dessas unidades.

Os resultados deste estudo são válidos apenas para mulheres que fizeram acompanhamento prénatal em unidades de saúde do SUS de baixo risco. Os desfechos encontrados devem ser melhores do que para a população geral, que engloba as gestantes sem acompanhamento pré-natal e dos setores privado e conveniado. A amostra deste trabalho foi

\section{Referências}

1. Manual Técnico Pré-natal e puerpério. Atenção qualificada e humanizada. Brasília, DF; Ministério da Saúde; 2006. 163 p.

2. Silveira MF, Santos IS, Barros AJD, Matijasevich, A, Barros FC, Victora CG. Aumento da prematuridade no Brasil: revisão de estudo de base populacional. Rev Saúde Pública. 2008; 42: 957-64.

3. Leal MC, Gama SGN, Ratto KMN, Cunha CB. Uso do índice de Kotelchuck modificado na avaliação da assistência pré-natal e sua relação com as características maternas e o peso do recém-nascido no município do Rio de Janeiro. Cad Saúde Pública. 2004; 20 (Suppl. 1): S6372 .

4. Covarrubias LO, Cáceres CW, Vázquez FA, Dávila Velázquez J, Eguiluz ME. Confounding effect of prematurity in the neonatal death associated with obstetric maternal morbidity. Ginecol Obstet Mex. 2009; 77: 277-81.

5. Vettore MV, Gama SG, Lamarca GA, Schilithz AO, Leal MC. Housing conditions as a social determinant of low birthweight and preterm low birthweight. Rev Saúde Pública. 2010; 44: 1021-31.

6. Orr ST, Reiter JP, James SA, Orr CA. Maternal health prior to pregnancy and preterm birth among urban, low income black women in Baltimore: the Baltimore Preterm Birth Study. Ethn Dis. 2012; 22: 85-9.

7. Sayres Jr WG. Preterm Labor. Am Fam Physician. 2010; 81: 477-84.

8. Goldenberg RL, Culhane JF, Iams JD, Romero R Epidemiology and causes of preterm birth. Lancet. 2008; 371: 75-84.

9. Gama SGNG, Szwarcwald CL, Leal MC. Experiência de gravidez na adolescência, fatores associados e resultados perinatais entre puérperas de baixa renda. Cad Saúde Pública. 2002; 18: 153-161. oriunda de gestantes atendidas em serviços de prénatal de baixo risco e portanto, caso fossem incluídas também as de alto risco, é provável que a proporção de grávidas com história de prematuridade fosse maior. Em virtude disso, possíveis associações entre passado de parto pré-termo e comorbidades maternas, tais como hipertensão arterial, diabetes e infecções, poderiam ser detectadas.

A proporção de pré-natal inadequado foi observada em um número significativo de gestantes e a presença da história de prematuridade não influenciou para que essas gestantes tivessem um pré-natal de melhor qualidade.

As unidades de saúde apresentavam problemas, segundo a percepção das gestantes, em relação ao modo de funcionamento, assim como as explicações fornecidas pelos profissionais de saúde sobre os riscos da prematuridade não corresponderam às suas expectativas.

10. Brasil. Ministério do Planejamento, Orçamento e Gestão Instituto Brasileiro de Geografia e Estatística (IBGE). Indicadores sociodemográficos e de saúde no Brasil. Brasília, DF; 2009.

11. Morgan-Ortiz F, Cinco-Sánchez A, Douriet-Marín FA, Báez-Barraza J, Muñoz-Acosta J, Osuna-Ramirez Sociodemographic and obstetric factors associated with preterm birth. Ginecol Obstet Mex. 2010; 78: 103-9.

12. Camargo RPS, Simões JA, Cecatti JG, Alves VMN, Faro S. Impacto do tratamento da vaginose bacteriana na prematuridade em gestantes brasileiras: uma coorte retrospectiva. São Paulo Med J. 2005; 123: 108-12.

13. Slager J, Lynne S. Treatment options and recommendations to reduce preterm births in women with short cervix. J Midwifery Womens Health. 2012; 57 (Suppl. 1): S12-8.

14. Wang Y, Tanbo T, Ellingsen L, Abyholm T, Henriksen T. Effect of pregestational maternal, obstetric and perinatal factors on neonatal outcome in extreme prematurity. Arch Gynecol Obstet. 2011; 284: 1381-7.

15. Zomignani AP, Zambelli HJL, Antonio MARG. Desenvolvimento cerebral em recém-nascidos prematuros. Rev Paul Pediatr. 2009; 27: 198-203.

16. Rio de Janeiro. Secretaria Municipal de Saúde. Indicadores de Saúde da Cidade do Rio de Janeiro por Área Programática (AP)- 1997 a 2008. [acesso em 21 mar 2013]. Disponível em: www.rio.rj.gov.br/web/smsdc/exibeconteudo?article-id $=156055$

17. Szwarcwald CL, Damacena GN. Amostras complexas em inquéritos populacionais: planejamento e implicações na análise estatística dos dados. Rev Bras Epidemiol. 2008; 11 (Suppl. 1): 38-45.

18. Kotelchuck M. An evaluation of the Kessner Adequacy of Prenatal Care Index and a proposed adequacy of prenatal care utilization index. Am J Public Health. 1994; 84: 141420 . 
19. Silva AMR, Almeida MF, Matsuo T, Soares DA. Fatores de risco para nascimentos pré-termo em Londrina, Paraná, Brasil. Cad Saúde Pública. 2009; 25: 2125-38.

20. Maloni JA. Antepartum bed rest for pregnancy complications: efficacy and safety for preventig preterm birth. Biol Res Nurs. 2010; 12: 106-24.

21. Schlüssel MM, Souza EB, Reichenheim ME, Kac G. Atividade física na gestação e desfechos da saúde maternoinfantil: uma revisão sistemática da literatura. Cad Saúde Pública. 2008; 24 (Suppl. 4): S531-44.

22. Andrade PC, Linhares JJ, Martinelli S, Antonini M, Lippi UG, Baracat FF. Resultados perinatais em grávidas com mais de 35 anos: estudo controlado. Rev Bras Ginecol Obstet. 2004; 26: 697-702.

23. Leal MC, Gama SGN, Cunha CB. Desigualdades raciais, sociodemográficas e na assistência ao pré-natal e ao parto, 1999-2001. Rev Saúde Pública. 2005; 39: 100-7.

24. Whitehead N, Helms K. Racial and ethnic differences in preterm delivery among low-risk women. Ethn Dis. 2010; 20: 261-6.

25. Beeckman K, Louckx F, Downe S, Putman K. The relationship between antenatal care and preterm birth: the importance of content of care. Eur J Public Health. 2013; 23: 366-71.

Recebido em 30 de março de 2012

Versão final apresentada em 21 de março 2013

Aprovado em 18 de abril de 2013
26. Assunção PL, Novaes HM, Alencar GP, Melo AS, Almeida MF. Factors associated with preterm birth in Campina Grande, Paraíba State, Brazil: a case-control study. Cad Saúde Pública. 2012; 28: 1078-90.

27. Carvalho MHB, Bittar RE, Gonzales M, Brizot ML, Zugaib M. Avaliação do risco para parto premturo espontâneo pelo comprimento do colo uterino no primeiro e segundo trimestre da gravidez. Rev Bras Ginecol Obstet. 2002; 24 : 463-8.

28. Garcia-Cavazos R, Colin-Valenzuela A, Espino y Sosa S. Alpha-fetoprotein as an early predictor of adverse perinatal outcome. Ginecol Obste Mex. 2010; 78: 268-74.

29. Couto ER, Couto E, Vian B, Gregório Z, Nomura ML, Zaccaria R, Passini Junior R. Quality of life, depression and anxiety among pregnant women with previous adverse pregnancy outcomes. São Paulo Med J. 2009; 127: 185-9.

30. Olusanya BO, Solanke OA. Perinatal outcomes associated with maternal hypertensive disorders of pregnancy in a developing country. Hypertens Pregnancy. 2012; 31: 12030 . 